\title{
From the Editor/
}

\section{Message de la rédactrice en chef ${ }^{1}$}

At Technostyle we are pleased to present another edition through the able efforts of a team of professional writing students at Douglas College. As one of the components of the Print Futures Program, two students, Ken Johnston and Susan Stacey, completed their practicum requirement by heading up a team of volunteer assistants. Technostyle is very fortunate to have such an excellent staff - they have done a superb job.

The contributing scholars to this issue have provided our readers with another collection of stimulating and informative articles. Linda Sanderson and Celine Beaudet discuss specific professional writing genres courtroom discourse and the annual report respectively. Deborah Begoray's ethnographic study takes us inside the collaborative writing process, and Pamela Russell, Jacqueline Bossé-Andrieu and Hélène Cajolet-Laganière report on survey research into the practices and genres of technical writing in French.

This edition of Technostyle also inaugurates our new "Notes" section of shorter pieces focused on teaching-related topics, both in the classroom (e.g. methods and evaluation) and in professional settings (e.g. consulting and workshopping). This first contribution focusses on placement testing in an academic context. Shaney Crawford reports on the methodology and results of a test used at Queen's University to identify ESL errors. Readers will also find in this issue a very informative textbook review by Lilita Rodman.

I would like to extend CATTW's appreciation to both contributors and editorial staff.

\section{Diana Wegner}

Nous sommes heureux de vous présenter ce nouveau numéro de Technostyle, réalisé grâce aux efforts d'une équipe d'étudiants en rédaction professionnelle au collège Douglas. Deux étudiants, Ken Johnston et Susan Stacey, ont complété leur stage, composante obligatoire du programme Print Futures, en dirigeant un groupe d'assistants bénévoles. Toute l'équipe a fait un excellent travail.

Nos lecteurs trouveront dans ce numéro un recueil d'articles stimulants et instructifs. Linda Sanderson et Céline Beaudet traitent de genres spécifiques à la rédaction professionnelle — soit le discours du tribunal et le rapport annuel. Létude ethnographique de Deborah Begoray

1. Traduit en français par Marie-Claire Chinniah 
explore le processus de rédaction en équipe, alors que Pamela Russell, Jacqueline Bossé-Andrieu et Hélène Cajolet-Laganière présentent les résultats d'une étude sur les pratiques et les genres de la rédaction technique et scientifique en français.

Dans ce numéro de Technostyle, nous inaugurons aussi notre rubrique "Considérations pédagogiques", consacrée à de courts articles sur des sujets reliés à l'enseignement, soit dans la salle de classe (ex: méthodologie et évaluation), soit dans le milieu professionnel (ex: consultations et ateliers). Pour cette "première", notre nouvelle rubrique porte sur les examens de classement dans un contexte universitaire. Le rapport de Shaney Crawford examine la méthodologie et les résultats d'un test utilisé à l'université Queen's pour identifier les erreurs fréquentes en anlglais langue seconde. Les lecteurs trouveront aussi dans ce numéro l'intéressante critique de texte de Lilita Rodman.

Au nom de l'ACPRTF, je tiens à remercier les auteurs et toute l'équipe de rédaction. 